Research Paper

\title{
Evaluating the Prognostic Value of ERCCl and Thymidylate Synthase Expression and the Epidermal Growth Factor Receptor Mutation Status in Adenocarcinoma Non-Small-Cell Lung Cancer
}

\author{
Chang-Sheng Lin ${ }^{1,2}$, Tu-Chen Liu ${ }^{3}$, Ji-Ching Lai ${ }^{4}$, Shun-Fa Yang1,5凶, Thomas Chang-Yao Tsao6, $7 凶$
}

1. Institute of Medicine, Chung Shan Medical University, Taichung, Taiwan;

2. Department of Chest Medicine, Show Chwan Memorial Hospital, Changhua, Taiwan;

3. Department of Chest Medicine, Cheng-Ching General Hospital, Taichung, Taiwan;

4. Research Assistant Center, ChangHua Show Chwan Health Care System, Changhua, Taiwan;

5. Department of Medical Research, Chung Shan Medical University Hospital, Taichung, Taiwan;

6. Division of Chest, Department of Internal Medicine, Chung Shan Medical University Hospital, Taichung, Taiwan;

7. School of Medicine, Chung Shan Medical University, Taichung, Taiwan.

$\triangle$ Corresponding authors: Thomas Chang-Yao Tsao, M.D., Ph.D. Division of Thoracic Medicine, Chung Shan University Hospital and Chung Shan Medical University, Taichung, Taiwan. Tel: +886-4-24730022 ext. 11020. Fax: +886-4-24759950. E-mail: his885889@gmail.com Or Shun-Fa Yang, PhD. Institute of Medicine, Chung Shan Medical University, Taichung, Taiwan. E-mail: ysf@csmu.edu.tw

(c) Ivyspring International Publisher. This is an open access article distributed under the terms of the Creative Commons Attribution (CC BY-NC) license (https://creativecommons.org/licenses/by-nc/4.0/). See http://ivyspring.com/terms for full terms and conditions.

Received: 2017.07.16; Accepted: 2017.10.11; Published: 2017.11.02

\begin{abstract}
The present study evaluated the prognostic value of the epidermal growth factor receptor (EGFR) mutation status, and excision repair cross-complementation group 1 (ERCCl) and thymidylate synthase (TS) expression following intercalated tyrosine kinase inhibitor (TKI) therapy and platinum- and pemetrexed-based chemotherapies (subsequent second-line treatment) for patients with adenocarcinoma non-small-cell lung cancer (AC-NSCLC). In total, 131 patients with AC-NSCLC were enrolled. The EGFR mutation status and ERCCl and TS expression were evaluated through direct DNA sequencing and immunohistochemical analyses, respectively. The EGFR mutation status and ERCCI and TS expression were the significant predictors of clinical outcomes. The EGFR mutation status was the main outcome predictor for overall survival (OS) benefits in the overall population. Further exploratory ERCCl and TS expression analyses were conducted to provide additional insights. Low TS expression was predictive of improved OS of patients with negative EGFR-mutated advanced AC-NSCLC, whereas high ERCCl expression resulted in poor OS in patients with positive EGFR-mutated advanced AC-NSCLC. TS and ERCCl expression levels were effective prognostic factors for negative and positive EGFR-mutated AC-NSCLC, respectively. In conclusion, the present results indicate that the EGFR mutation status and TS and ERCCI expression can be used as the predictors of OS after subsequent second-line treatments for AC-NSCLC.
\end{abstract}

Key words: non-small-cell lung cancer, EGFR mutation status, ERCC1 expression, TS protein expression, tyrosine-kinase inhibitor, chemotherapy.

\section{Introduction}

Lung cancer is the leading cause of cancer-related mortality worldwide, particularly in highly developed counties [1]. It has been classified histologically into two major groups: small-cell lung cancer and non-small-cell lung cancer (NSCLC). Adenocarcinoma, squamous cell carcinoma, bronchioalveolar carcinoma, and large cell carcinoma are the subtypes of NSCLC. More than $80 \%$ of NSCLC cases are of the adenocarcinoma subtype (AC-NSCLC), whose incidence rate has steadily increased over the past decade. Approximately $65 \%$ of these cases are diagnosed at an advanced stage [2]. The standard therapies for lung adenocarcinoma include epidermal growth factor receptor (EGFR) 
mutation targeted therapy and conventional chemotherapy, such as platinum- and pemetrexed-based chemotherapies.

Several biomarkers have been used to predict treatment responses, such as the EGFR mutation status for targeted therapy or the excision repair cross-complementation group 1 (ERCC1) and thymidylate synthase (TS) expression for conventional chemotherapy [3-8]. Mutations in the EGFR tyrosine kinase (TK) domain were first reported in 2004 [9], and were found in approximately $50 \%$ of Asian patients with NSCLC [10]. With the introduction of personalized treatments, molecular target agents, such as EGFR-TK inhibitors (TKIs) have been used in clinical practice for the treatment of advanced NSCLC. Many randomized controlled trials of patients with active EGFR mutations have demonstrated the superiority of the TKI therapy over conventional cytotoxic drug treatments in terms of progression-free survival, the objective response rate, and overall survival (OS) [11-15]. In the Iressa Pan-Asia study (iPASS), the first-line EGFR-TKIs treatment was found to have a significant OS benefit to unselected patients with NSCLC, compared with those who received conventional chemotherapy [16]. Therefore, EGFR-TKIs have been used as the standard first-line treatment for patients with NSCLC, particularly for AC-NSCLC with active EGFR mutations. However, the subgroup analysis in the iPASS revealed that conventional chemotherapy exhibited surprisingly good efficacy in the active EGFR mutation group [16]. Therefore, conventional cytotoxic drug-based chemotherapies have become the standard second-line treatment for patients with lung adenocarcinoma after TKI therapy failure.

Platinum-based chemotherapy is a DNA damage therapy for NSCLC. ERCC1 is a rate-limiting factor of the nucleotide excision repair (NER) system, which is involved in the repair of cytotoxic platinum-DNA adducts [17]. Therefore, high ERCC1 expression has been associated with resistance to platinum-based chemotherapy in various tumors, including NSCLC [18-20], whereas ERCC1-negative NSCLC has been associated with higher survival rates after platinum-based chemotherapy [21]. TS is an enzyme that catalyzes the conversion of deoxyuridine monophosphate to deoxythymidine monophosphate. Pemetrexed is a folate antimetabolite that is chemically similar to folic acid and inhibits enzyme activity, and thus contributes to DNA damage. High TS expression is involved in a resistance mechanism and may be a predictive biomarker of pemetrexed sensitivity in NSCLC [22, 23]. Pemetrexed-based chemotherapy is used as a first-line treatment and has been currently approved as the first-line treatment for patients with nonsquamous cell histology in combination with platinum [24]. However, the association of ERCC1 and TS expression with the prognosis of AC-NSCLC with different EGFR mutation statuses is yet to be elucidated.

To understand the clinical significance of these biomarkers, including the EGFR mutation status and TS and ERCC1 expression, in terms of treatment outcomes, 131 patients with AC-NSCLC who received the TKI therapy and pemetrexed- and platinum-based chemotherapies were evaluated. The correlation between treatment response as well as OS and the three biomarkers, was also investigated.

\section{Materials and Methods}

\section{Patients}

In total, 131 patients with AC-NSCLC were enrolled. First, patient clinical information, including age, sex, smoking status, and the order of receiving TKI therapy and conventional chemotherapies, was collected. The EGFR mutation status of all patients was evaluated before initiation of the TKI therapy or chemotherapy, and therapy was provided according to their EGFR mutation status. Specifically, patients with positive EGFR-mutated AC-NSCLC received the TKI therapy as the first-line treatment and pemetrexed- and platinum-based chemotherapies as the subsequent second-line treatment after TKI therapy failure. By contrast, patients with negative EGFR-mutated AC-NSCLC were administered the pemetrexed- and platinum-based chemotherapies as the first-line treatment followed by EGFR-TKI targeted therapy as the second-line treatment. To evaluate patients' OS, chest computed tomography was performed every 3 months. The treatment response was evaluated according to the guidelines of Response Evaluation Criteria in Solid Tumors (version 1.1) [25]. OS was defined as the period from the initiation date of TKI therapy or chemotherapy to the date of death or last patient contact. This study was approved by the Institutional Review Board of Show Chwan Memorial Hospital (IRB No. 1030110), and all experiments, procedures, and methods were performed in accordance with the IRB-approved guidelines and regulations.

\section{EGFR mutation test}

All tumor DNA samples were obtained and extracted from paraffin-embedded blocks prepared on initial diagnosis. The DNA sequences of exons 18-21 of EGFR were determined by direct sequencing of the polymerase chain reaction (PCR) product as previously described $[26,27]$. EGFR positive mutation results were confirmed by sequencing an independent PCR product. In the present study, the absence of 
EGFR mutation was defined as "negative," whereas all EGFR alteration types, including L858R and exon 19 deletion, were defined as "positive."

\section{Immunohistochemical analysis for TS and ERCCI expression}

An immunohistochemical (IHC) analysis was performed to detect ERCC1 and TS expression by using monoclonal ERCC1 and TS antibodies (SC-17809 and SC-33679, respectively, Santa Cruz Biotechnology, Inc., Santa Cruz, CA, USA), respectively. The protocol of IHC analysis was according to the methods described by Lin et al. [28].

\section{Scoring of TS and ERCCI expression}

The IHC analysis results of ERCC1 and TS expression were examined, and the scores were assessed by board-certified pathologists. Every slide was scored according to the intensity of nuclear and cytoplasmic staining for ERCC1 and TS expression, respectively. For ERCC1 expression, nuclear staining in $0 \%-50 \%$ and $>50 \%$ of cancerous cells was scored as low and high immunostaining, respectively [29]. For TS expression, cytoplasmic staining in $0 \%-30 \%$ and $>30 \%$ of cancerous cells was scored as low and high immunostaining, respectively [30].

\section{Statistics}

A chi-squared test was performed to assess the correlation between clinical parameters and the EGFR mutation status and ERCC1 and TS expression; specifically, the Fischer exact test was used because of the small sample size. Survival curves were plotted using the Kaplan-Meier method, and statistical significance was determined using the log-rank test. Cox proportional hazards regression analysis and all statistical analyses were performed using SPSS for Windows (version 12; SPSS, Inc., Chicago, IL, USA). A two-sided $\mathrm{p}$ value of $<0.05$ was considered statistically significant.

Table 1. Population characteristics among adenocarcinomas non-small cell lung cancer tested.

\begin{tabular}{ll}
\hline Characteristic & Frequency, $\mathrm{n}(\%)$ \\
\hline Mean age, years (standard deviation) & $65.87(11.62)$ \\
Female & $57(43.5)$ \\
Male & $74(56.5)$ \\
Stage at time of test & \\
I, II & $29(22.1)$ \\
III, IV & $102(77.9)$ \\
Smoking Status & \\
Never & $85(64.9)$ \\
Current smoker or ever smoked & $46(35.1)$ \\
\hline
\end{tabular}

\section{Results}

\section{Population characteristics}

In total, 131 patients with AC-NSCLC were included in this study (Table 1). The mean age of the patients was $65.87 \pm 11.62$ years, and $56.5 \%$ of these patients were men. In total, $102(77.9 \%)$ of the patients had advanced stage (III or IV) AC-NSCLC, and 29 $(22.1 \%)$ of the patients had early stage (I or II) AC-NSCLC. Of these patients, $64.9 \%$ had never smoked.

\section{Relationship between biomarkers (EGFR mutation status and ERCCI and TS expression) and the clinical parameters of AC-NSCLC}

Figure 1 shows the representative IHC analysis results of ERCC1 and TS expression, and Table 2 presents patient characteristics according to the EGFR mutation status and IHC analysis results. Of the 131 patients with AC-NSCLC, 61 (47\%) had at least one mutation in the EGFR TK domain. Two mutation types were detected at the in-frame exon 19 deletion in 36 patients $(27 \%)$, and the exon 21 point mutation L858R was found in 25 patients (19\%). No other EGFR mutation types were observed in our study. Two significant associations were observed between the somatic mutations of EGFR and the clinical parameters, including female sex $(p<0.001)$ and the never-smoking status $(\mathrm{p}<0.001$; Table 2$)$. ERCC1 and TS expression was localized to the nucleus (Figure 1b) and cytoplasm (Figure 1d), respectively. Most patients in this study had low ERCC1 (63\%) and TS (66\%) expression, and ERCC1 expression and sex exhibited a significant association (Table 2; $p=0.002$ for sex). Moreover, TS expression was significantly higher in men $(\mathrm{p}=0.022)$ and smokers $(\mathrm{p}=0.011)$. The relationship between ERCC1 and TS expression and the EGFR mutation status was validated (Table 3). Overall, positive EGFR mutation was highly correlated with low ERCC1 and TS expression $(p<$ 0.001 for both).

\section{Correlations between biomarkers (EGFR mutation status and ERCCI and TS expression) and clinical responses to the second-line treatment}

In the 58 patients with advanced AC-NSCLC and follow-up OS, the effects of the biomarkers on OS after the second-line treatment were validated by a univariate Kaplan-Meier analysis (Table 4). The clinical parameters, including young age $(p=0.028)$, female sex $(p<0.001)$, the never-smoking status $(p<$ $0.001)$, positive EGFR mutation $(p<0.001)$, and low ERCC1 and TS expression (both $p<0.001$ ) (Figures 2A 
and $2 \mathrm{~B}$, respectively), were the prognostic predictors of AC-NSCLC. Moreover, the Cox proportional hazards regression analysis revealed that young patients $(p=0.001)$ and those with a positive EGFR mutation ( $p<0.001)$, low ERCC1 expression ( $p=$ $0.007)$, and low TS expression $(\mathrm{p}=0.006)$ had a significantly improved OS, compared with older patients and those with a negative EGFR mutation, high ERCC1 expression, and high TS expression, respectively (Table 5). According to Table 4, the IHC

A

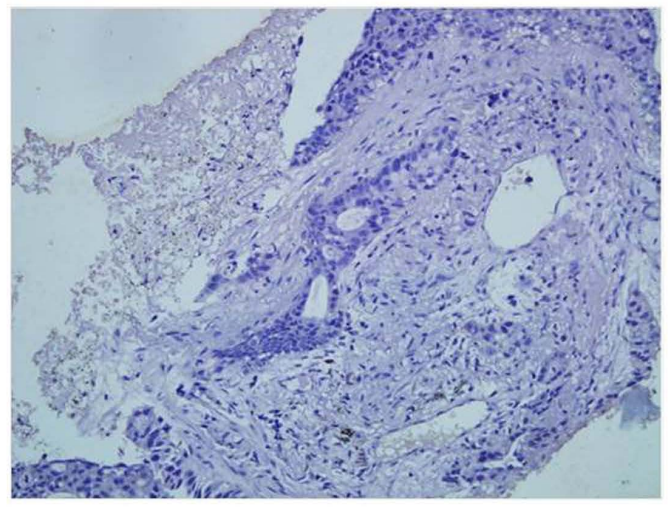

$\mathrm{C}$

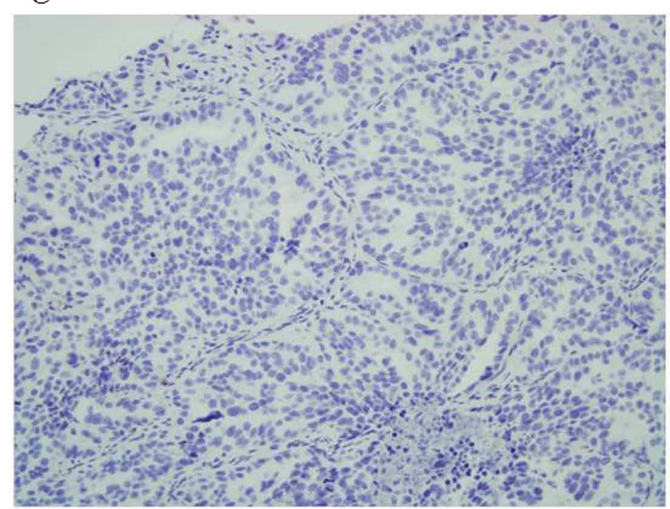

analysis results validated the effects of ERCC1 and TS expression on the OS of the EGFR mutation status. Figures 2C and 2D present the Kaplan-Meier survival curves. Notably, older patients and those with high ERCC1 (Figure 2C) and TS (Figure 2D) expression had lower OS than did younger patients and those with low ERCC1 and TS expression in the negative EGFR mutation AC-NSCLC group $(\mathrm{p}=0.003, \mathrm{p}=0.025$, and $p=0.001$, respectively).

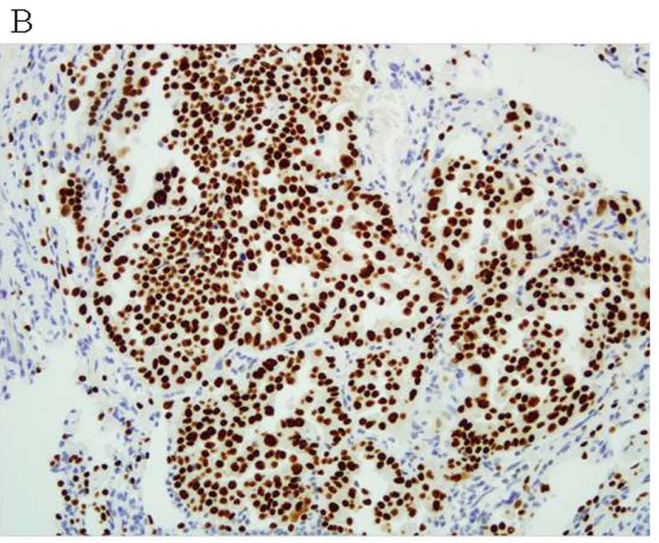

D

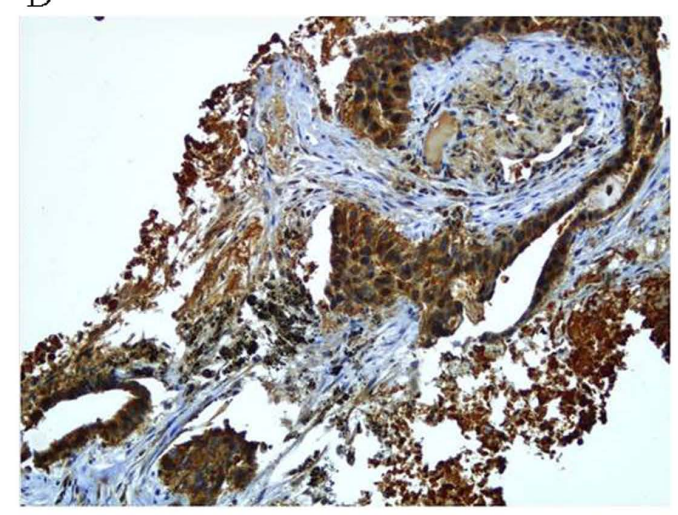

Figure 1. Representative of ERCC1 and TS protein immunostainings in paraffin sections of AC-NSCLC tumors. A. negative ERCC1 immunostaining (100X). B. positive ERCC1. immunostaining (100X). C. negative TS immunostaining (100X). D. positive TS. immunostaining (100X).

Table 2. Association of clinic parameters with EGFR mutation, ERCCl and TS immunostainings.

\begin{tabular}{|c|c|c|c|c|c|c|c|c|c|}
\hline \multirow[t]{2}{*}{ Characteristic } & \multicolumn{3}{|c|}{ EGFR mutation } & \multicolumn{3}{|l|}{ ERCC1 } & \multicolumn{3}{|l|}{ TS } \\
\hline & Unfound (\%) & Positive (\%) & $P$ & Low (\%) & High (\%) & $P$ & Low $(\%)$ & High (\%) & $P$ \\
\hline \multicolumn{10}{|l|}{ Age (years) } \\
\hline$<65$ & $30(56)$ & $24(44)$ & 0.684 & $36(67)$ & $18(33)$ & 0.42 & $36(68)$ & $18(32)$ & 0.959 \\
\hline$\geqq 65$ & $40(52)$ & $37(48)$ & & $46(60)$ & $31(40)$ & & $51(66)$ & $26(34)$ & \\
\hline \multicolumn{10}{|l|}{ Gender } \\
\hline Female & $19(33)$ & $38(67)$ & $<0.001$ & $44(77)$ & $13(23)$ & 0.002 & $44(77)$ & $13(23)$ & 0.022 \\
\hline Male & $51(69)$ & $23(31)$ & & $38(51)$ & $36(49)$ & & $43(58)$ & $31(42)$ & \\
\hline \multicolumn{10}{|l|}{ Stage $\#$} \\
\hline I, II & $12(41)$ & $17(59)$ & 0.14 & $20(69)$ & $9(31)$ & 0.422 & $21(72)$ & $8(28)$ & 0.438 \\
\hline III, IV & $58(57)$ & $44(43)$ & & $62(61)$ & $40(39)$ & & $66(65)$ & $36(35)$ & \\
\hline \multicolumn{10}{|l|}{ Smoking Status\# } \\
\hline Never & $29(34)$ & $56(66)$ & $<0.001$ & $58(68)$ & $27(32)$ & 0.07 & $63(74)$ & $22(26)$ & 0.011 \\
\hline Current smoker or ever smoked & $41(89)$ & $5(11)$ & & $24(52)$ & $22(48)$ & & $24(52)$ & $22(48)$ & \\
\hline
\end{tabular}

Abbreviations: EGFR, epidermal growth factor receptor; ERCC1, Excision repair cross-complementing group 1; TS, Thymidylate synthase.

EGFR mutation: including L858R and exon 19 deletion.

\#Fisher's exact test. 
The Cox proportional hazards regression analysis indicated that the risk ratio (RR) of TS expression and age were 4.34- and 3.491-fold ( $\mathrm{p}=$ $0.019,95 \%$ confidence interval $[\mathrm{CI}]=1.273-14.79$ and $\mathrm{p}=0.028,95 \% \mathrm{CI}=1.143-10.66$, respectively) in the negative EGFR mutation AC-NSCLC group. In the positive EGFR-mutated AC-NSCLC group, the clinical parameters and ERCC1 and TS expression, as well as patients' OS, were determined through univariate analysis. Older patients and those with high ERCC1 expression had significantly poorer OS outcomes, compared with younger patients and those with low ERCC1 expression ( $p=0.043$ and $p=0.002$, respectively) (Figures $2 \mathrm{E}$ and $2 \mathrm{~F}$, Table 4). TS expression was not a significant predictor in the positive EGFR-mutated AC-NSCLC group (Figure 2F, $\mathrm{p}=0.117)$. The RRs of ERCC1 expression, age, and sex were 14.84-, 3.161-, and 2.99-fold, respectively ( $\mathrm{p}=$ $0.001,95 \% \mathrm{CI}=2.986-73.75 ; \mathrm{p}=0.012,95 \% \mathrm{CI}=$ 1.292-7.733; and $p=0.020,95 \% C I=1.190-7.517$, respectively) in the positive EGFR-mutated
AC-NSCLC group (Table 5). Notably, in the negative EGFR-mutated AC-NSCLC group, the patients with high TS expression had a 4.34-fold higher RR than did those with low TS expression. In the positive EGFR-mutated AC-NSCLC group, the patients with high ERCC1 expression had a 14.84-fold higher RR than those with low ERCC1 expression. Therefore, TS and ERCC1 expression levels are effective predictors for negative and positive EGFR-mutated AC-NSCLC, respectively.

Table 3. Relationships between EGFR mutation and ERCC1, TS immunostainings.

\begin{tabular}{llll}
\hline Characteristic & \multicolumn{3}{l}{ EGFR Mutation } \\
\cline { 2 - 4 } & Unfound (\%) & Positive (\%) & $P$ \\
\hline ERCC1 & $28(34)$ & $54(66)$ & $<0.001$ \\
Low & $42(86)$ & $7(14)$ & \\
High & & & \\
TS & $34(39)$ & $53(61)$ & $<0.001$ \\
Low & $36(82)$ & $8(18)$ & \\
High &
\end{tabular}

Table 4. Univariate analysis of influences of clinical characteristics on overall survival of follow-up NSCLC patients.

\begin{tabular}{|c|c|c|c|c|c|c|c|c|c|}
\hline \multirow[t]{2}{*}{ Characteristics } & \multicolumn{3}{|c|}{ Follow-up cases } & \multicolumn{3}{|c|}{ Unfound EGFR mutation } & \multicolumn{3}{|c|}{ Positive EGFR mutation } \\
\hline & $\begin{array}{l}\text { No. of } \\
\text { cases }\end{array}$ & $\begin{array}{l}\text { Median survival } \\
\text { (Months) }\end{array}$ & Log-rank* & $\begin{array}{l}\text { No. of } \\
\text { cases }\end{array}$ & $\begin{array}{l}\text { Median survival } \\
\text { (Months) }\end{array}$ & Log-rank* & $\begin{array}{l}\text { No. of } \\
\text { cases }\end{array}$ & $\begin{array}{l}\text { Median survival } \\
\text { (Months) }\end{array}$ & Log-rank* \\
\hline \multicolumn{10}{|l|}{ Age (years) } \\
\hline$<65$ & 21 & 22 & 0.028 & 9 & 11 & 0.003 & 12 & 28 & 0.043 \\
\hline$\geqq 65$ & 37 & 17 & & 17 & 6 & & 20 & 22 & \\
\hline \multicolumn{10}{|l|}{ Gender } \\
\hline Female & 26 & 22 & $<0.001$ & 5 & 11 & 0.266 & 21 & 28 & 0.065 \\
\hline Male & 32 & 10 & & 21 & 7 & & 11 & 22 & \\
\hline \multicolumn{10}{|l|}{ Smoking status } \\
\hline Never smoked & 39 & 21 & $<0.001$ & 10 & 10 & 0.181 & 29 & 26 & 0.491 \\
\hline Current smoker or ever smoked & 19 & 7 & & 16 & 6 & & 3 & 26 & \\
\hline \multicolumn{10}{|l|}{ EGFR mutation } \\
\hline Unfound & 26 & 9 & $<0.001$ & & & & & & \\
\hline Positive & 32 & 26 & & & & & & & \\
\hline \multicolumn{10}{|l|}{ ERCC1 immunostaining } \\
\hline Low & 37 & 22 & $<0.001$ & 8 & 11 & 0.025 & 29 & 27 & 0.002 \\
\hline High & 21 & 7 & & 18 & 6 & & 3 & 15 & \\
\hline \multicolumn{10}{|l|}{ TS immunostaining } \\
\hline Low & 38 & 22 & $<0.001$ & 11 & 12 & 0.001 & 27 & 27 & 0.117 \\
\hline High & 20 & 9 & & 15 & 6 & & 5 & 20 & \\
\hline
\end{tabular}

*. Log-rank $p$-values for categorical variables were statistically analyzed by Kaplan-Meier test.

Table 5. Cox regression analysis of various potential prognostic factors in NSCLC patients

\begin{tabular}{|c|c|c|c|c|c|c|c|c|c|c|}
\hline \multirow[t]{2}{*}{ Variables } & \multirow{2}{*}{$\begin{array}{l}\text { Unfavorable } \\
\text { /Favorable }\end{array}$} & \multicolumn{3}{|c|}{ Follow-up cases } & \multicolumn{3}{|c|}{ Unfound EGFR mutation } & \multicolumn{3}{|c|}{ Positive EGFR mutation } \\
\hline & & RR & $P^{1}$ & $95 \% \mathrm{CI}$ & RR & $P^{2}$ & $95 \% \mathrm{CI}$ & RR & $P^{2}$ & $95 \% \mathrm{CI}$ \\
\hline EGFR mutation & unfound/positive & 0.115 & $<0.001$ & $0.043-0.307$ & & & & & & \\
\hline ERCC1 & high/low & 3.643 & 0.007 & $1.432-9.267$ & 0.239 & 0.113 & $0.814-7.012$ & 14.84 & 0.001 & $2.986-73.75$ \\
\hline TS & high/low & 2.773 & 0.006 & $1.345-5.721$ & 4.340 & 0.019 & $1.273-14.79$ & 1.950 & 0.232 & $0.652-5.830$ \\
\hline Age & $\geqq 65 /<65$ & 2.922 & 0.001 & $1.540-5.546$ & 3.491 & 0.028 & $1.143-10.66$ & 3.161 & 0.012 & $1.292-7.733$ \\
\hline Gender & male/female & 2.072 & 0.066 & $0.953-4.506$ & 0.943 & 0.935 & $0.229-3.877$ & 2.990 & 0.020 & $1.190-7.517$ \\
\hline Smoking status & $\begin{array}{l}\text { current or ever smoked/ } \\
\text { never smoked }\end{array}$ & 1.331 & 0.499 & $0.581-3.047$ & 1.784 & 0.314 & $0.578-5.510$ & 0.747 & 0.683 & $0.184-3.027$ \\
\hline
\end{tabular}

1. Adjusted for EGFR mutation, ERCC1 immunostaining, TS immunostaining, age, gender and smoking status.

2. Adjusted for ERCC1 immunostaining, TS immunostaining, age, gender and smoking status. 
A

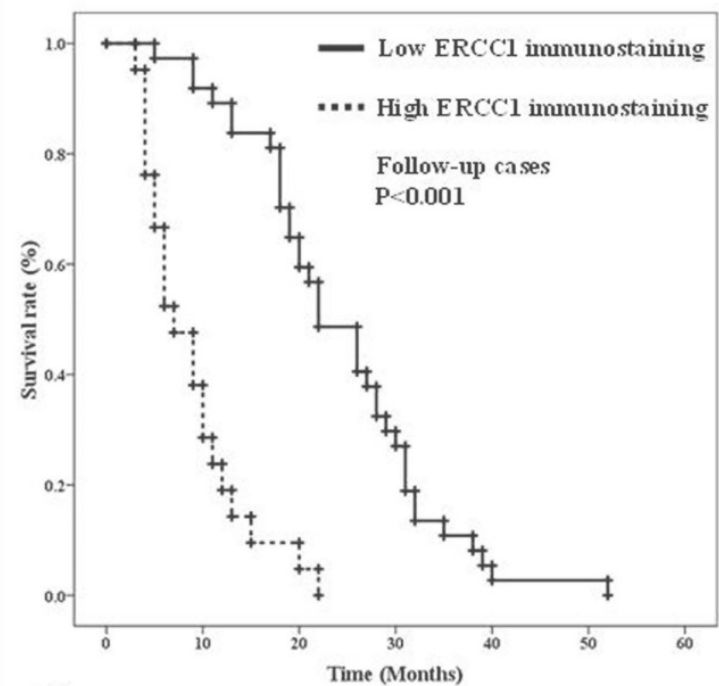

C

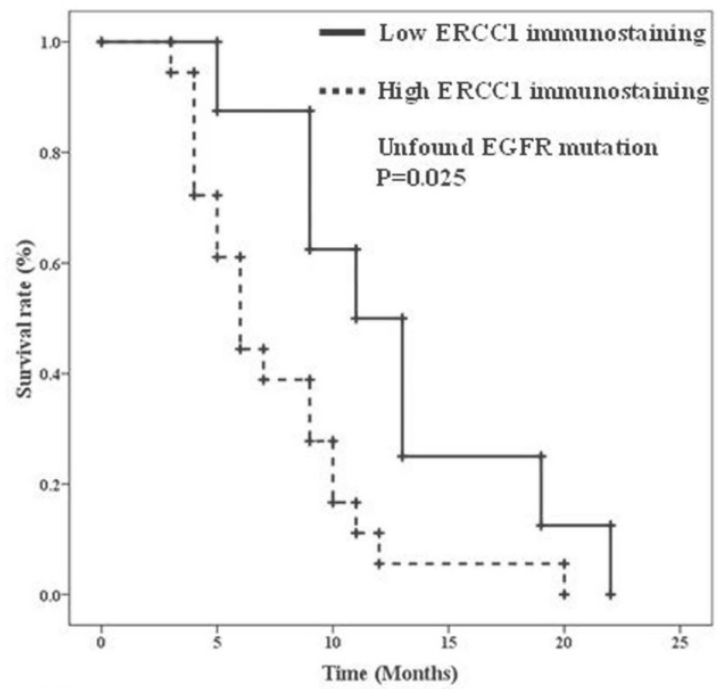

E

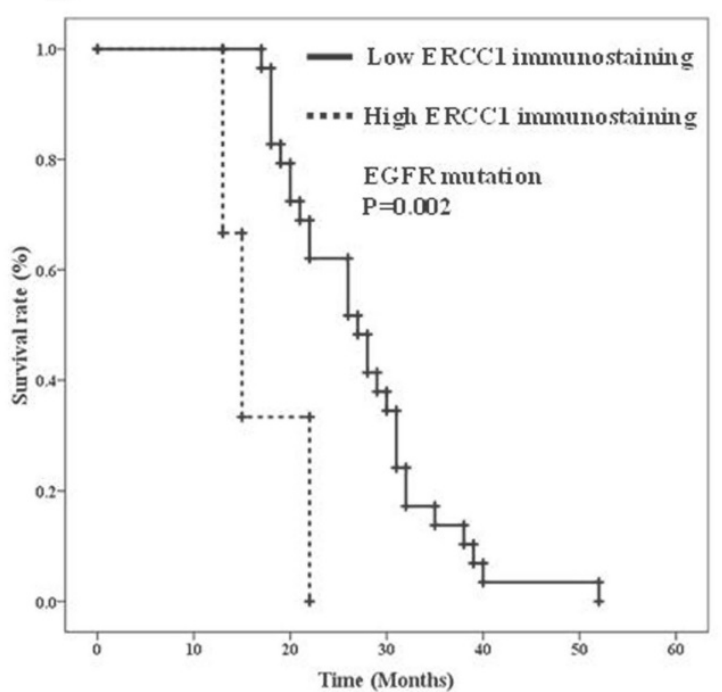

B

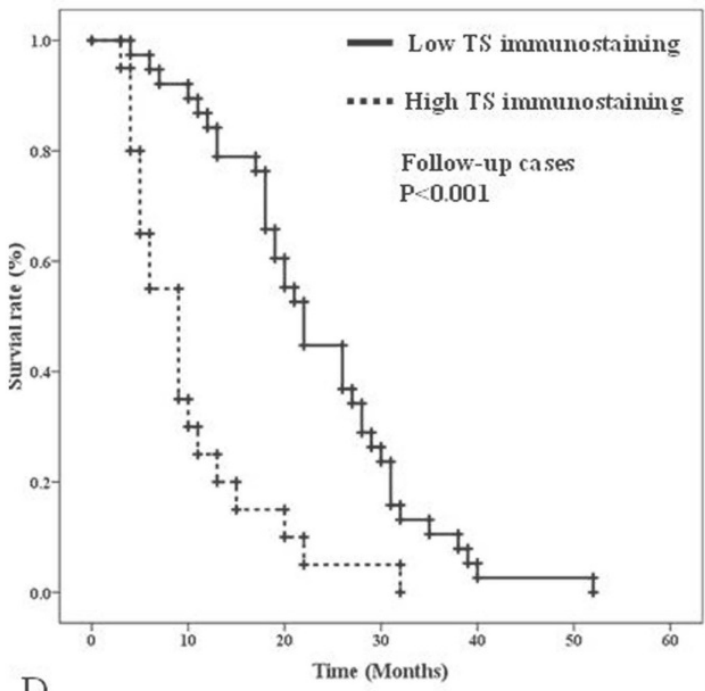

D

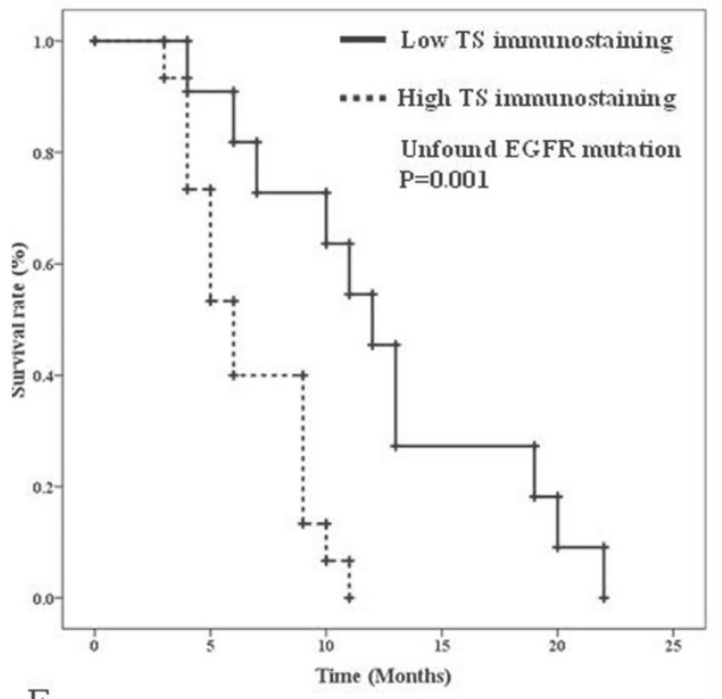

F

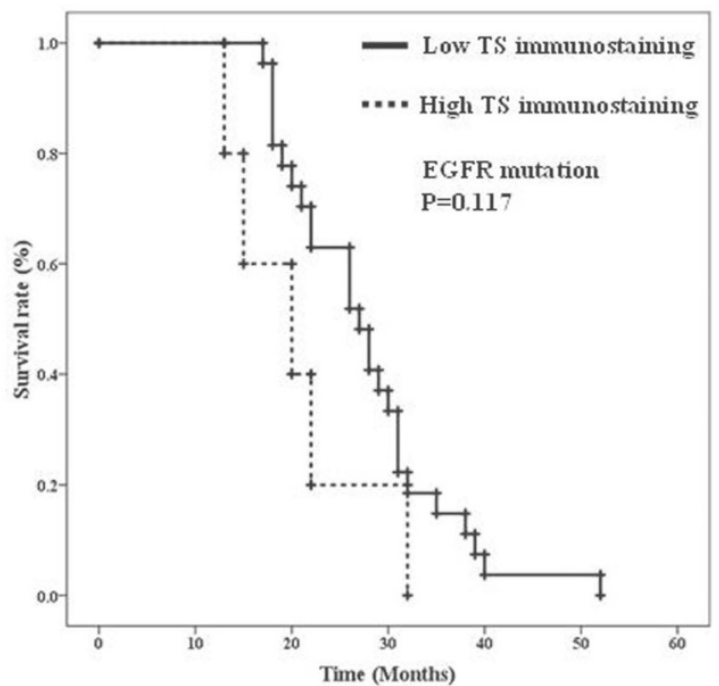

Figure 2. Overall survival analysis of ERCCI and TS immunostainings in AC-NSCLC stratified by EGFR mutation status. A. Kaplan-Meier survival curves of ERCCl immunostaining in AC-NSCLC. B. Kaplan-Meier survival curves of TS immunostaining in AC-NSCLC. C. Kaplan-Meier survival curves of ERCC1 immunostaining in unfound EGFR mutation AC-NSCLS. D. Kaplan-Meier survival curves of TS immunostaining in unfound EGFR mutation AC-NSCLS. E. Kaplan-Meier survival curves of ERCC1 immunostaining in positive EGFR mutation AC-NSCLS. F. Kaplan-Meier survival curves of TS immunostaining in positive EGFR mutation AC-NSCLS. 


\section{Discussion}

In the present retrospective study, 58 patients with AC-NSCLC were administrated TKI therapy and pemetrexed- and platinum-based chemotherapies. The correlation between OS and their EGFR mutation status and TS and ERCC1 expression was then analyzed. A positive EGFR mutation and low TS and ERCC1 expression were the most favorable predictors of OS, with the EGFR mutation status being the strongest predictor of AC-NSCLC after adjustment for TS and ERCC1 expression. The newly discovered interlinked biomarkers were associated with the treatment response after the second-line treatment, and led to the prudent assessment of a personalized therapy strategy for AC-NSCLC.

Nevertheless, several of the results merit further discussion. First, TKI therapy (gefitinib or erlotinib) was provided as the first-line treatment for 32 patients with AC-NSCLC and somatic mutations at the EGFR TK domain, such as exon 19 and 21. After TKI therapy failure, pemetrexed- and platinum-based chemotherapies were administered. For the patients with negative EGFR-mutated AC-NSCLC, pemetrexed- and platinum-based chemotherapies were provided as the first-line treatment, and the TKI therapy was administered after chemotherapy failure. Active EGFR mutations have been confirmed as the predictive biomarkers for EGFR-TKI targeted therapy in patients with advanced NSCLC. Additionally, pemetrexed- and platinum-based chemotherapies results in the formation of DNA monoadducts and interstrand crosslinks [31]. The elimination of DNA adducts is mainly dependent on the NER pathway [32]. However, until now, the prognostic value of ERCC1 and TS expression for AC-NSCLC was unclear. Most studies have revealed that ERCC1 expression is correlated with resistance to chemotherapy. In one, adjuvant cisplatin-based therapy achieved prolonged OS in ERCC1-negative tumors but not in ERCC1-positive tumors [33]. Elsewhere, patients with low or median TS and ERCC1 expression had survival benefits after pemetrexed- and platinum-based chemotherapies [34, 35]. Additionally, the DNA repair biomarker, ERCC1, was determined to have a significant predictive value in squamous cell carcinomas [36]. However, the readout ERCC1 IHC data were unreliable to consider ERCC1 as a prognostic marker of NSCLC in another study [37]. He et al. [38] also reports that chemotherapy based on ERCC1 expression did not have significant impact on disease-free survival of patients with NSCLC. TS expression, rather than ERCC1 expression, was found to be the only independent prognostic factor in malignant pleural mesothelioma [39]. Furthermore, TS expression was higher in nonadenocarcinomas (non-AC-NSCLC) than in AC-NSCLC, and high TS expression was a powerful prognostic predictor of poor outcomes in resected AC-NSCLC [30].

With the exception of the EGFR mutation status, which served as a major prognostic biomarker for OS (Table 4), we analyzed ERCC1 and TS expression subgroups according to the EGFR mutation status. The patients in the subgroups with negative EGFR mutation and high ERCC1 and TS expression had significantly decreased OS. Moreover, notable findings were obtained for the subgroups with and without EGFR mutation. High TS expression can be considered a prognostic factor in negative EGFR-mutated AC-NSCLC, whereas high ERCC1 expression might be a poor prognostic factor in positive EGFR-mutated AC-NSCLC. In short, ERCC1 and TS expression levels can be used as clinical outcome predictors for positive and negative EGFR-mutated AC-NSCLC, respectively. These findings and the clinical significance of the EGFR mutation status and ERCC1 and TS expression must be validated with further analyses.

In conclusion, despite the small sample size of the present study, our findings indicate that the EGFR mutation status is the main biomarker for treatment outcomes following second-line treatment. Our study suggests that the EGFR mutation status and ERCC1 and TS expression are useful biomarkers for evaluating the OS of patients with positive or negative EGFR-mutated AC-NSCLC. However, this observation is merely a hypothesis at present, and requires further validation.

\section{Competing Interests}

The authors have declared that no competing interest exists.

\section{References}

1. Siegel RL, Miller KD, Jemal A. Cancer statistics, 2016. CA Cancer J Clin. 2016; 66: 7-30.

2. William WN Jr, Lin HY, Lee JJ, Lippman SM, Roth JA, Kim ES. Revisiting stage IIIB and IV non-small cell lung cancer: analysis of the surveillance, epidemiology, and end results data. Chest. 2009; 136: 701-9.

3. Lynch TJ, Bell DW, Sordella R, Gurubhagavatula S, Okimoto RA, Brannigan $\mathrm{BW}$, et al. Activating mutations in the epidermal growth factor receptor underlying responsiveness of non-small-cell lung cancer to gefitinib. N Engl J Med. 2004; 350: 2129-39.

4. Gazdar AF, Minna JD. Inhibition of EGFR signaling: All mutations are not created equal. PLoS Med. 2005; 2: e377

5. Hirsch FR. EGFR: a prognostic and/or a predictive marker? J Thorac Oncol. 2006; 1: 395-7.

6. $\mathrm{Li} \mathrm{J}, \mathrm{Li}$ ZN, Yu LC, Bao QL, Wu JR, Shi SB, et al. Association of expression of MRP1, BCRP, LRP and ERCC1 with outcome of patients with locally advanced non-small cell lung cancer who received neoadjuvant chemotherapy. Lung Cancer. 2010; 69: 116-22.

7. Azuma K, Sasada T, Kawahara A, Takamori S, Hattori S, Ikeda J, et al. Expression of ERCC1 and class III beta-tubulin in non-small cell lung cancer patients treated with carboplatin and paclitaxel. Lung Cancer. 2009; 64: 326-33.

8. Bepler G, Sommers KE, Cantor A, Li X, Sharma A, Williams C, et al. Clinical efficacy and predictive molecular markers of neoadjuvant gemcitabine and 
pemetrexed in resectable non-small cell lung cancer. J Thorac Oncol. 2008; 3: $1112-8$

9. Pao W, Miller V, Zakowski M, Doherty J, Politi K, Sarkaria I, et al. EGF receptor gene mutations are common in lung cancers from "never smokers" and are associated with sensitivity of tumors to gefitinib and erlotinib. Proc Natl Acad Sci U S A. 2004; 101: 13306-11.

10. Hirsch FR, Bunn PA Jr. EGFR testing in lung cancer is ready for prime time. Lancet Oncol. 2009; 10: 432-3.

11. Maemondo M, Inoue A, Kobayashi K, Sugawara S, Oizumi S, Isobe H, et al. Gefitinib or chemotherapy for non-small-cell lung cancer with mutated EGFR. N Engl J Med. 2010; 362: 2380-8.

12. Mitsudomi T, Morita S, Yatabe Y, Negoro S, Okamoto I, Tsurutani J, et al. Gefitinib versus cisplatin plus docetaxel in patients with non-small-cell lung cancer harbouring mutations of the epidermal growth factor receptor (WJTOG3405): an open label, randomised phase 3 trial. Lancet Oncol. 2010; 11: $121-8$.

13. Rosell R, Carcereny E, Gervais R, Vergnenegre A, Massuti B, Felip E, et al. Erlotinib versus standard chemotherapy as first-line treatment for European patients with advanced EGFR mutation-positive non-small-cell lung cancer (EURTAC): a multicentre, open-label, randomised phase 3 trial. Lancet Oncol. 2012; 13:239-46.

14. Zhou $\mathrm{C}, \mathrm{Wu}$ YL, Chen G, Feng J, Liu XQ, Wang C, et al. Erlotinib versus chemotherapy as first-line treatment for patients with advanced EGFR mutation-positive non-small-cell lung cancer (OPTIMAL, CTONG-0802): a multicentre, open-label, randomised, phase 3 study. Lancet Oncol. 2011; 12: $735-42$

15. Shepherd FA, Rodrigues Pereira J, Ciuleanu T, Tan EH, Hirsh V, Thongprasert $\mathrm{S}$, et al. Erlotinib in previously treated non-small-cell lung cancer. N Engl J Med. 2005; 353: 123-32.

16. Mok TS, Wu YL, Thongprasert S, Yang CH, Chu DT, Saijo N, et al. Gefitinib or carboplatin-paclitaxel in pulmonary adenocarcinoma. N Engl J Med. 2009; 361: 947-57.

17. Reed E. Nucleotide excision repair and anti-cancer chemotherapy. Cytotechnology 1998; 27: 187-201.

18. Rosell R, Lord RV, Taron M, Reguart N. DNA repair and cisplatin resistance in non-small-cell lung cancer. Lung Cancer. 2002; 38:217-27.

19. Olaussen KA, Dunant A, Fouret P, Brambilla E, André F, Haddad V, et al. DNA repair by ERCC1 in non-small-cell lung cancer and cisplatin-based adjuvant chemotherapy. N Engl J Med 2006; 355: 983-91.

20. Postel-Vinay S, Soria JC. ERCC1 as Predictor of Platinum Benefit in Non-Small-Cell Lung Cancer. J Clin Oncol. 2017; 35: 384-6.

21. Chen S, Zhang J, Wang R, Luo X, Chen $\mathrm{H}$. The platinum-based treatments for advanced non-small cell lung cancer, is low/negative ERCC1 expression better than high/positive ERCC1 expression? A meta-analysis. Lung Cancer. 2010; 70: 63-70.

22. Takezawa K, Okamoto I, Okamoto W, Takeda M, Sakai K, Tsukioka S, et al. Thymidylate synthase as a determinant of pemetrexed sensitivity in non-small cell lung cancer. Br J Cancer 2011; 104: 1594-601.

23. Chen CY, Chang YL, Shih JY, Lin JW, Chen KY, Yang CH, et al. Thymidylate synthase and dihydrofolate reductase expression in non-small cell lung carcinoma: the association with treatment efficacy of pemetrexed. Lung Cancer 2011; 74: 132-8.

24. Scagliotti GV, Parikh P, von Pawel J, Biesma B, Vansteenkiste J, Manegold C, et al. Phase III study comparing cisplatin plus gemcitabine with cisplatin plus pemetrexed in chemotherapy-naïve patients with advanced-stage non-small cell lung cancer. J Clin Oncol 2008; 26: 3543-51.

25. Eisenhauer EA, Therasse P, Bogaerts J, Schwartz LH, Sargent D, Ford R, et al. New response evaluation criteria in solid tumours: revised RECIST guideline (version 1.1). Eur J Cancer 2009; 45: 228-47.

26. Liu TC, Hsieh MJ, Liu MC, Chiang WL, Tsao TC, Yang SF. The Clinical Significance of the Insulin-Like Growth Factor-1 Receptor Polymorphism in Non-Small-Cell Lung Cancer with Epidermal Growth Factor Receptor Mutation. Int J Mol Sci. 2016; 17: E763.

27. Liu TC, Hsieh MJ, Wu WJ, Chou YE, Chiang WL, Yang SF, et al. Association between survivin genetic polymorphisms and epidermal growth factor receptor mutation in non-small-cell lung cancer. Int J Med Sci. 2016; 13: 929-35

28. Lin CS, Liu TC, Lee MT, Yang SF, Tsao TC. Independent Prognostic Value of Hypoxia-inducible Factor 1-alpha Expression in Small Cell Lung Cancer. Int J Med Sci. 2017; 14: 785-90

29. Mok T, Ladrera G, Srimuninnimit V, Sriuranpong V, Yu CJ, Thongprasert S, et al. Tumor marker analyses from the phase III, placebo-controlled, FASTACT-2 study of intercalated erlotinib with gemcitabine/platinum in the first-line treatment of advanced non-small-cell lung cancer. Lung Cancer. 2016; 98:1-8.

30. Kaira K, Ohde Y, Nakagawa K, Okumura T, Murakami H, Takahashi T, et al. Thymidylate synthase expression is closely associated with outcome in patients with pulmonary adenocarcinoma. Med Oncol. 2012; 29: 1663-72.

31. Lawley PD, Phillips DH. DNA adducts from chemotherapeutic agents. Mutat Res 1996; 355: 13-40.

32. Hoeijmakers JH. Genome maintenance mechanisms for preventing cancer. Nature 2001; 411: 366-74.

33. Olaussen KA, Dunant A, Fouret P, Brambilla E, André F, Haddad V, et al. DNA repair by ERCC1 in non-small-cell lung cancer and cisplatin-based adjuvant chemotherapy. N Engl J Med. 2006; 355: 983-91.
34. Fujii T, Toyooka S, Ichimura K, Fujiwara Y, Hotta K, Soh J, et al. ERCC1 protein expression predicts the response of cisplatin-based neoadjuvant chemotherapy in non-small-cell lung cancer. Lung Cancer. 2008; 59: 377-84.

35. Liu D, Nakashima N, Nakano J, Tarumi S, Matsuura N, Nakano T, et al. Customized Adjuvant Chemotherapy Based on Biomarker Examination May Improve Survival of Patients Completely Resected for Non-small-cell Lung Cancer. Anticancer Res. 2017; 37: 2501-7.

36. Pierceall WE, Olaussen KA, Rousseau V, Brambilla E, Sprott KM, Andre F, et al. Cisplatin benefit is predicted by immunohistochemical analysis of DNA repair proteins in squamous cell carcinoma but not adenocarcinoma: theranostic modeling by NSCLC constituent histological subclasses. Ann Oncol. 2012; 23: 2245-52.

37. Wislez M, Barlesi F, Besse B, Mazières J, Merle P, Cadranel J, et al. Customized adjuvant phase II trial in patients with non-small-cell lung cancer: IFCT-0801 TASTE. J Clin Oncol. 2014; 32: 1256-61.

38. He YW, Zhao ML, Yang XY, Zeng J, Deng QH, He JX. Prognostic value of ERCC1, RRM1, and TS proteins in patients with resected non-small cell lung cancer. Cancer Chemother Pharmacol. 2015. 75: 861-7.

39. Righi L, Papotti MG, Ceppi P, Billè A, Bacillo E, Molinaro L, et al. Thymidylate synthase but not excision repair cross-complementation group 1 tumor expression predicts outcome in patients with malignant pleural mesothelioma treated with pemetrexed-based chemotherapy. J Clin Oncol. 2010; 28: 1534-9. 